\title{
Expansion of Criteria for Liver Transplantation in Hepatocellular Carcinoma: Better Patient Selection or a Slippery Slope?
}

\author{
Adam C. Yopp, $\mathrm{MD}^{1}$, Jorge A. Marrero, $\mathrm{MD}^{2}$, and Amit G. Singal, $\mathrm{MD}^{2}$ \\ ${ }^{1}$ Division of Surgical Oncology, Department of Surgery, University of Texas Southwestern Medical Center, Dallas, TX; \\ ${ }^{2}$ Division of Digestive and Liver Diseases, Department of Medicine, University of Texas Southwestern Medical Center, \\ Dallas, TX
}

Hepatocellular carcinoma (HCC) is the third leading cause of cancer-related deaths worldwide and one of the leading causes of death in patients with cirrhosis. ${ }^{1}$ In appropriately selected patients, orthotopic liver transplantation (OLT) provides 5-year overall survival rates approaching $70 \%$ and is the only therapy that offers both a cure for the cancer and the underlying liver cirrhosis. Prior to publication of the Milan criteria by Mazzaferro et al. in 1996, early results of OLT for HCC were dismal due to the high rate of recurrence and poor overall outcome. Adherence to the Milan criteria, a solitary tumor $\leq 5 \mathrm{~cm}$ or with three or less tumors each $\leq 3 \mathrm{~cm}$ with no evidence of vascular invasion or extrahepatic metastasis, has led to excellent post-transplant survival and forms the framework to award priority for deceased donor allocation in the US utilizing the model for end-stage liver disease (MELD) score. $^{2}$

Although adaptation of the Milan criteria has facilitated survival rates that approach those of non-HCC patients, some believe strict adherence to these criteria may preclude the benefits of OLT from patients with larger tumors. This has led to the development of several proposals for expanded transplant criteria, including the University of California San Francisco (UCSF) and up-to-seven criteria, whereupon selected patients beyond the Milan criteria could still be eligible for transplantation. ${ }^{3,4}$ Although initially designed based on retrospective pathological explant data, the UCSF criteria, but not the up-to-seven criteria,

(C) Society of Surgical Oncology 2017

First Received: 24 January 2017;

Published Online: 17 March 2017

A. C. Yopp, MD

e-mail: adam.yopp@utsouthwestern.edu have been validated in studies using a priori pre-transplant radiographic imaging, with 5-year post-transplantation survival rates approaching $70 \% .^{5}$ Recently, expanded criteria adding surrogates of tumor biology to tumor size and number was proposed by the University of Toronto group. The extended Toronto criteria (ETC) allows OLT for HCC provided there is no evidence of cancer-related symptoms, extrahepatic spread, vascular invasion, or poor differentiation on pre-transplant biopsy. Patients who exceeded the Milan criteria but satisfied the ETC achieved 5-year survival rates of $70 \%$ in the original single-institution experience, although these results have not yet been externally validated. ${ }^{6}$ A follow-up study by the same group demonstrated that 5-year survival rates for patients outside of the Milan criteria but within the UCSF criteria and the ETC were numerically lower but not significantly inferior than patients within the Milan criteria (68 vs. 76\%; $p=0.07)^{7}$

Aravinthan et al., the authors of the original ETC studies, examined their single institution, retrospective experience for OLT among 96 patients with HCC outside the UCSF criteria but within the ETC over a 15-year period. ${ }^{8}$ The hypothesis of the authors is that better long-term survival could be achieved with OLT than locoregional or systemic therapy options, where historical 3-year survival rates are approximately $30 \%$ and 5-year survival rates are typically less than $5 \%{ }^{9,10}$ The authors found OLT afforded these patients 5 -year survival rates of $66 \%$ and concluded OLT is a viable treatment option for patients presenting with HCC outside of the UCSF criteria but within the ETC. Although only based on 69 of 96 patients (28\% dropout from the waitlist) who underwent OLT, the overall survival and dropout rates were similar to other series evaluating expanded criteria beyond the Milan criteria. 
The main difference between the ETC and other expanded criteria, including the UCSF and the up-to-seven criteria, is inclusion of a preoperative biopsy to determine HCC differentiation as a surrogate of tumor biology. The degree of differentiation correlates to post-transplant survival and recurrence rates, with poorly differentiated tumors faring significantly worse than well or moderately differentiated tumors. ${ }^{11}$ This is an important distinction as tumor burden alone is an imperfect surrogate for tumor biology, with substantial variation in natural history and treatment responsiveness observed between patients with the same degree of tumor burden. However, what is less clear is the best way to assess tumor biology. Many markers of tumor biology are currently not available pretransplant (e.g. histology), not validated (e.g. circulating tumor cells), or insufficiently accurate (e.g. $\alpha$-fetoprotein). Although the ETC used pre-treatment tumor biopsy to exclude poorly differentiated tumors from OLT, the feasibility of adopting this approach in a more widespread fashion is questionable. There may be reluctance among patients and providers given the risks of bleeding, injury to other organs, and, most notably, tumor seeding. Even when performed, HCC displays intratumoral heterogeneity, limiting reliable grading of differentiation by biopsy. ${ }^{12}$ In fact, in the current study, nearly $10 \%$ of patients initially graded as well or moderately differentiated tumors on pre-treatment biopsies can be upgraded to poorly differentiated on final explant histology. Other investigators have also demonstrated this lack of concordance between pre-treatment grading on biopsy and final HCC grading on larger post-surgical specimens, calling into question its ability to reliably and accurately guide patient selection for OLT. ${ }^{12}$

Beyond the debate between specific criteria, the adoption of expanded criteria for OLT as a general policy poses a dilemma in the face of limited organ availability. The persistent shortage of donor organs means the importance of deriving maximum benefit from this valuable community resource has to be taken into account. ${ }^{13}$ Since the advent of the MELD allocation system for cadaveric donor organs, approximately $25 \%$ of all liver transplants in the US are carried out for HCC, despite patients with HCC accounting for only approximately $10 \%$ of patients on the waiting list. ${ }^{14}$ Although it is clear that patients with favorable HCC tumor biology undoubtedly benefit from OLT compared with locoregional and systemic options, the adoption of more liberal criteria must be weighed with harms (decreased organ availability and increased mortality) for non-HCC patients on the waiting list. The harms of expanding selection criteria typically outweigh the benefits when 5-year post-transplant survival rates fall below $61 \%$, but this is dependent on waitlist times and regional and local center MELD scores for non-HCC patients in terms of MELD. ${ }^{15}$ A major limitation of many of the studies examining expanded criteria for OLT in HCC patients is the absence of accounting for waitlist mortality and dropout rate for patients without $\mathrm{HCC}$ who are in essence 'competing' with HCC patients for a scarce resource. Until this analysis is described in a head-to-head fashion in organ allocation policies, it is difficult to advocate for an extended criteria in a vacuum.

By the authors' own account, widespread adoption of the ETC would dramatically increase the number of HCC patients on the waitlist. It is unclear how this increased demand would be managed and how it would affect those waiting for transplant without HCC. The authors suggest increasing the use of living donor liver transplants (LDLT) could fill the void of increased demand without harming patients on the waiting list; however, the stable number of LDLTs performed in the US over the last decade seems to belie this claim. ${ }^{14}$

Recent changes in organ allocation policy may render this dilemma a moot point. A recent United Network for Organ Sharing (UNOS) policy change also requires a minimum of 6 months before $\mathrm{HCC}$ patients can receive MELD exception points, with a cap of 35 points. This change may improve patient selection through observation of tumor biology following bridging therapy and avoid the need for biopsy and its associated complications. This concept has been confirmed in multiple studies where a shorter waitlist time is associated with worse post-transplant outcomes. This new policy of 'HCC Cap and Delay', which was meant to level the playing field for transplantation of non-HCC patients, will ultimately cause harm to these patient by increasing the waitlist mortality compared with HCC patients. The MELD Exceptions and Enhancements Subcommittee is also considering a change to the MELD exception policy in which patients with tumor burden exceeding the Milan criteria would be eligible for inclusion in a downstaging protocol. This includes patients with one lesion $>5$ and $\leq 8 \mathrm{~cm}$, those with two to three lesions each $<5 \mathrm{~cm}$ and total diameter $\leq 8 \mathrm{~cm}$, and those with four to five lesions each $<3 \mathrm{~cm}$ and total diameter $\leq 8 \mathrm{~cm}$. Patients must complete locoregional therapy and subsequently meet requirements for T2 HCC. Similar to the mandatory 6 month waiting period, this policy change aims to select patients with good tumor biology and lower risk of post-transplant recurrence.

It is clear that the Milan criteria provided a necessary framework to make liver transplantation for HCC a mainstream therapy for these patients, and the adoption of more liberal expanded criteria is now possible. However, the push beyond the Milan criteria must be tempered by the greater societal need to allocate a scarce resource to the majority of patients who need OLT, i.e. those without HCC who have no viable treatment alternatives. The use of surrogates to measure tumor biology may allow us to 
accurately select HCC patients who would benefit the most from OLT regardless of tumor burden. Until this happens, OLT criteria for HCC should not be expanded.

\section{REFERENCES}

1. Are C, Rajaram S, Are M, et al. A review of global cancer burden: trends, challenges, strategies, and a role for surgeons. J Surg Oncol. 2013;107:221-6.

2. Mazzaferro V, Regalia E, Doci R, et al. Liver transplantation for the treatment of small hepatocellular carcinomas in patients with cirrhosis. N Engl J Med. 1996;334:693-9.

3. Yao FY, Ferrell L, Bass NM, et al. Liver transplantation for hepatocellular carcinoma: expansion of the tumor size limits does not adversely impact survival. Hepatology. 2001;33:1394-403.

4. Mazzaferro V, Llovet JM, Miceli R, et al. Predicting survival after liver transplantation in patients with hepatocellular carcinoma beyond the Milan criteria: a retrospective, exploratory analysis. Lancet Oncol. 2009;10:35-43.

5. Yao FY, Xiao L, Bass NM, et al. Liver transplantation for hepatocellular carcinoma: validation of the UCSF-expanded criteria based on preoperative imaging. Am J Transplant. 2007; 7:2587-96.

6. DuBay D, Sandroussi C, Sandhu L, et al. Liver transplantation for advanced hepatocellular carcinoma using poor tumor differentiation on biopsy as an exclusion criterion. Ann Surg. 2011; 253:166-72.

7. Sapisochin G, Goldaracena N, Laurence JM, et al. The extended Toronto criteria for liver transplantation in patients with hepatocellular carcinoma: a prospective validation study. Hepatology. 2016;64:2077-88.

8. Aravinthan AD, Bruni SG, Doyle AC, et al. Liver Transplantation is a preferable alternative to palliative therapy for selected patients with advanced hepatocellular carcinoma. Ann Surg Oncol. 2017. doi:10.1245/s10434-017-5789-3.

9. Llovet JM, Real MI, Montana X, et al. Arterial embolisation or chemoembolisation versus symptomatic treatment in patients with unresectable hepatocellular carcinoma: a randomised controlled trial. Lancet. 2002;359:1734-9.

10. Llovet JM, Ricci S, Mazzaferro V, et al. Sorafenib in advanced hepatocellular carcinoma. N Engl J Med. 2008;359:378-90.

11. Jonas S, Bechstein WO, Steinmuller T, et al. Vascular invasion and histopathologic grading determine outcome after liver transplantation for hepatocellular carcinoma in cirrhosis. Hepatology. 2001;33:1080-6.

12. Pawlik TM, Gleisner AL, Anders RA, et al. Preoperative assessment of hepatocellular carcinoma tumor grade using needle biopsy: implications for transplant eligibility. Ann Surg. 2007;245:435-42.

13. Institute of Medicine Committee on Organ Procurement and Transplantation Policy. Organ procurement and transplantation: assessing current policies and the potential impact of the DHHS final rule. Washington: National Academy Press; 1999. pp. 1-38.

14. Kim WR, Lake JR, Smith JM, et al. Liver. Am J Transplant. 2016;16:69-98.

15. Volk ML, Vijan S, Marrero JA. A novel model measuring the harm of transplanting hepatocellular carcinoma exceeding Milan criteria. Am J Transplant. 2008;8:839-46. 